\title{
A Signal Decomposition Method for Ultrasonic Guided Wave Generated from Debonding Combining Smoothed Pseudo Wigner-Ville Distribution and Vold-Kalman Filter Order Tracking
}

\author{
Junhua Wu, Xinglin Chen, and Zheshu Ma \\ School of Automotive and Traffic Engineering, Nanjing Forestry University, Nanjing, Jiangsu 210037, China \\ Correspondence should be addressed to Junhua Wu; bjtime13@126.com
}

Received 15 May 2017; Accepted 3 August 2017; Published 14 September 2017

Academic Editor: Michele Palermo

Copyright (C) 2017 Junhua Wu et al. This is an open access article distributed under the Creative Commons Attribution License, which permits unrestricted use, distribution, and reproduction in any medium, provided the original work is properly cited.

Carbon fibre composites have a promising application future of the vehicle, due to its excellent physical properties. Debonding is a major defect of the material. Analyses of wave packets are critical for identification of the defect on ultrasonic nondestructive evaluation and testing. In order to isolate different components of ultrasonic guided waves (GWs), a signal decomposition algorithm combining Smoothed Pseudo Wigner-Ville distribution and Vold-Kalman filter order tracking is presented. In the algorithm, the time-frequency distribution of GW is first obtained by using Smoothed Pseudo Wigner-Ville distribution. The frequencies of different modes are computed based on summation of the time-frequency coefficients in the frequency direction. On the basis of these frequencies, isolation of different modes is done by Vold-Kalman filter order tracking. The results of the simulation signal and the experimental signal reveal that the presented algorithm succeeds in decomposing the multicomponent signal into monocomponents. Even though components overlap in corresponding Fourier spectrum, they can be isolated by using the presented algorithm. So the frequency resolution of the presented method is promising. Based on this, we can do research about defect identification, calculation of the defect size, and locating the position of the defect.

\section{Introduction}

Carbon fibre composite is widely used in modern industry, such as aerospace domain and military products, because of its high strength and light weight. At present, such a material has been generalized to automotive industry, obviously reducing the weight of automobile. Debonding defect is a major defect of the carbon fibre composites. A great number of investigations of the nondestructive evaluation and testing (NDE/NDT) have done research for this type of defect [1-5].

Currently, ultrasonic guided wave (GW) testing has emerged as a popular NDE/NDT technique. The method can estimate the location, severity, and type of defects. Successful applications of defect identification of carbon fibre composites have been done $[3,6,7]$. However, dispersion effects and noise make ultrasonic testing waves as multicomponent signals, which results in that it is difficult to do NDE/NDT with raw testing waves. Therefore, isolating different components of GW and obtaining the corresponding time-frequency distributions (TFD) are vital for the inspection of the defect.

A number of scholars have done investigations about signal processing methods of GWs. Kercel et al. [8] used Bayesian parameter estimates to isolate multiple modes in GW signals collected from laser ultrasonic testing on a manufacturing assembly line. Cai et al. [9] provided a timedistance domain transform (TDDT) method to interpret the dispersion of Lamb waves, which can result in high spatial resolution images of damage areas. Rizzo and di Scalea utilized Discrete Wavelet Transform (DWT) to extract wavelet domain features for enhanced defect characterization in multiwire strand structures [10]. Gangadharan et al. presented a time reversal technique using GWs to detect damage in an aluminum plate, and good results were achieved [11]. The wavelet analysis is widely used [12-17] in domains; many 
successful applications of wavelet transform (WT) for GW signals have been done. Li et al. [14] proposed a combined method employing empirical mode decomposition (EMD) and wavelet analysis to attain good time resolution of the response signals. Paget et al. [15] proposed a new damagedetection technique based on WT with a new basis. Yu et al. [16] used the techniques of statistical averaging to reduce global noise and discrete wavelet denoising using a Daubechies wavelet to remove local high-frequency disturbances. Y. Y. Kim and E.-H. Kim [17] evaluated the effectiveness of WT analysis for studying the wave dispersion.

EMD, which can isolate adaptively different components, was proposed by Huang in 1998 [18]. At present, many investigations of theory and application have been done [1924]. Li et al. [14], Osegueda et al. [20], and Salvino et al. [22] used EMD to process GW signals in plate structures. However, the frequency resolution of EMD is a limitation. Reference [25] reveals that when the ratio between a relatively low frequency and a relatively high frequency is greater than 0.75 , two components of a signal cannot be separated.

In 1993, Vold and Leuridan [26] proposed Vold-Kalman filter order tracking (VKF_OT) for the estimation of a single order component. In 1997, they [27] derived a scheme to simultaneously estimate multiple components. Instantaneous frequency of the isolated component is a necessary prior knowledge for VKF_OT. Therefore, we introduce Smoothed Pseudo Wigner-Ville distribution (SPWVD), which can remove the cross-term in frequency direction and time direction of the time-frequency panel, to get instantaneous frequencies of isolated components. We present a signal decomposition method for ultrasonic GWs combining VKF_OT and SPWVD in this paper.

The rest of this paper is organized as follows. Section 2 presents the theories of Smoothed pseudo Wigner-Ville distribution and Vold-Kalman filter order tracking. The principle of algorithm is illustrated in Section 3. Section 4 provides an illustration of the presented method. The details of the experiment are described in Section 5. Section 6 shows the application of the presented algorithm to the experimental signals. Finally, Section 7 concludes.

\section{Smoothed Pseudo Wigner-Ville Distribution and Vold-Kalman Filter Order Tracking}

2.1. Smoothed Pseudo Wigner-Ville Distribution. Wigner-Ville distribution has a fine time-frequency resolution and can reach the low boundary of Heisenberg uncertainty principle. It is defined as [28]

$$
\operatorname{WVD}_{s}(t, f)=\int_{-\infty}^{\infty} s\left(t+\frac{\tau}{2}\right) s\left(t-\frac{\tau}{2}\right) e^{-j 2 \pi f \tau} d \tau
$$

However, for multicomponent signals, it suffers from inevitable interference of cross-terms. SPWVD can remove it in frequency direction and time direction of the timefrequency panel. And the formula of SPWVD is as follows [28]:

$$
\begin{aligned}
\operatorname{SPW}_{s}(n, \Theta) & =\sum_{k=-L+1}^{L-1}|h(k)| \\
& \sum_{l=M+1}^{M-1} g(l) s(n+l+k) s^{*}(n+l-k) e^{-j 2 k \Theta},
\end{aligned}
$$

where $g(l)$ and $h(k)$ are smoothing window functions in time direction and frequency direction, respectively. $s$ is an analyzed signal, and $n$ and $\Theta$ are time variable and frequency variables, respectively. The time resolution and frequency resolution of SPWVD are promising. Moreover, no interference is in the representation.

2.2. Vold-Kalman Filter Order Tracking. Isolation of different modes is important for defect identification by ultrasonic guided waves. On this basic, we can locate the defect and evaluate the defect size. Therefore, VKF_OT is employed to separate wave packages.

In this paper, the angular-displacement VKF_OT techniques are adapted. The method is used to obtain the tracked components by minimizing the energy of errors for both the structural and data equations by mean of one of the least squares approaches [29].

The $k$ th order component can be defined as

$$
f_{k}(t)=a_{k}(t) \theta_{k}(t)+a_{-k}(t) \theta_{-k}(t),
$$

where $a_{k}(t)$ is the complex envelope and $a_{-k}(t)$ is the complex conjugate of $a_{k}(t)$ to make $f_{k}(t)$ a real waveform. It is noted that $\theta_{k}(t)$ is a carrier wave and defined as

$$
\theta_{k}(t)=\exp \left(k i \int_{0}^{t} \omega(u) d u\right),
$$

where $d u$ is the speed of the reference axle and $\int_{0}^{t} \omega(u) d u$ is the elapsed angular displacement. The discrete form of (4) can then be written as

$$
\theta_{k}(n)=\exp \left(k i \sum_{m=0}^{n} \omega(m) \Delta T\right) .
$$

2.2.1. The Structural Equation. As the tracked component $f_{k}(t)$ can be written as (3), where the envelope $a_{k}(t)$ needs to be computed. Generally, $a_{k}(t)$ fulfills [29]

$$
\frac{d^{S} a_{k}(t)}{d t^{s}}=\psi_{k}(t),
$$

where $\psi_{k}(t)$ is a higher-degree term in $a_{k}(t)$. The corresponding discrete forms can be expressed,

$$
\nabla^{S} a_{k}(n)=\psi_{k}(n),
$$

where $\nabla$ is the difference operator, the index $s$ is the differentiation order, and $\psi_{k}(n)$ physically is a combination of other spectral components and additional measurement noise. 
2.2.2. The Data Equation. A measured signal $y(n)$ can be taken as a combination of several components, $f_{k}(t)$, and measurement noise,

$$
y(n)=\sum_{k \in j} a_{k}(n) \theta_{k}(n)+\xi(n),
$$

where the integral number $j(= \pm 1, \pm 2, \pm 3, \ldots$, and/or $\pm K)$ is the order of spectral components to be tracked and $\xi(n)$ is unwanted spectral components and measurement errors. Each component $a_{k}(n)$ of interest modulates with a carrier wave $\theta_{k}(n)$.

2.2.3. Calculation of the Tracked Component $f$. Let $s=2$ and let data length be $N$; then the calculation matrix form can be expressed as [29]

$$
\begin{gathered}
{\left[\begin{array}{cccccccc}
-2 & 1 & 0 & 0 & 0 & \cdots & 0 & 0 \\
1 & -2 & 1 & 0 & 0 & \cdots & 0 & 0 \\
0 & 1 & -2 & 1 & 0 & \cdots & 0 & 0 \\
\vdots & \vdots & \vdots & \vdots & \vdots & \vdots & \vdots & \vdots \\
0 & 0 & 0 & 0 & 0 & \cdots & -2 & 1
\end{array}\right]\left[\begin{array}{c}
a_{k}(1) \\
a_{k}(2) \\
a_{k}(3) \\
\vdots \\
a_{k}(N)
\end{array}\right]} \\
=\left[\begin{array}{c}
\psi_{k}(1) \\
\psi_{k}(2) \\
\psi_{k}(3) \\
\vdots \\
\psi_{k}(N)
\end{array}\right] .
\end{gathered}
$$

To simultaneously track multiple orders and spectral components such as resonance, it can be extended to all order components of interest as well. Let

$$
\begin{aligned}
& \overleftrightarrow{M}=\left[\begin{array}{cccccccc}
-2 & 1 & 0 & 0 & 0 & \cdots & 0 & 0 \\
1 & -2 & 1 & 0 & 0 & \cdots & 0 & 0 \\
0 & 1 & -2 & 1 & 0 & \cdots & 0 & 0 \\
\vdots & \vdots & \vdots & \vdots & \vdots & \vdots & \vdots & \vdots \\
0 & 0 & 0 & 0 & 0 & \cdots & -2 & 1
\end{array}\right] \\
& \overleftrightarrow{A}=\left[\begin{array}{c}
a_{k}(1) \\
a_{k}(2) \\
a_{k}(3) \\
\vdots \\
a_{k}(N)
\end{array}\right] \\
& \overleftrightarrow{Z}=\left[\begin{array}{l}
\tilde{\psi}_{k}(1) \\
\tilde{\psi}_{k}(2) \\
\widetilde{\psi}_{k}(3) \\
\vdots \\
\widetilde{\psi}_{k}(N)
\end{array}\right]
\end{aligned}
$$

and then (9) becomes

$$
\left[\begin{array}{ccccccc}
\overleftrightarrow{M} & 0 & 0 & 0 & \cdots & 0 & 0 \\
0 & \overleftrightarrow{M} & 0 & 0 & \cdots & 0 & 0 \\
0 & 0 & \overleftrightarrow{M} & 0 & \cdots & 0 & 0 \\
\vdots & \vdots & \vdots & \vdots & \vdots & \vdots & \vdots \\
0 & 0 & 0 & 0 & \cdots & 0 & \overleftrightarrow{M}
\end{array}\right] \overleftrightarrow{A}=\overleftrightarrow{Z}
$$

where elements $\tilde{a}_{k}$ in the matrix $\overleftrightarrow{A}$ are column vectors with a length $N$, which is the $k$ th order component; $\widetilde{\psi}_{k}$ are error vectors with a dimension $N \times 1$; and $M$ is a matrix with a dimension $N \times N$.

The terms with negative indexes in (8) assure $f_{k}(t)$ to be a real waveform. $\tilde{y}$ is the measured signal with a length of $N$, $\xi$ an error vector with dimension $N \times 1$, and $\overleftrightarrow{B}_{k}$ consists of carrier signals, as

$$
\overleftrightarrow{B}_{k}=\left[\begin{array}{ccccc}
\theta_{k}(1) & 0 & 0 & \cdots & 0 \\
0 & \theta_{k}(2) & 0 & \cdots & 0 \\
0 & 0 & \theta_{k}(3) & \cdots & 0 \\
\vdots & \vdots & \vdots & \vdots & \vdots \\
0 & 0 & 0 & \cdots & \theta_{k}(N)
\end{array}\right] \overleftrightarrow{A}=\overleftrightarrow{Z}
$$

Thus, (8) can be rewritten as

$$
\tilde{y}-\left[\begin{array}{lllll}
\widetilde{B}_{1} & \widetilde{B}_{2} & \widetilde{B}_{3} & \cdots & \widetilde{B}_{k}
\end{array}\right]\left[\begin{array}{c}
\tilde{a}_{1} \\
\tilde{a}_{2} \\
\tilde{a}_{3} \\
\vdots \\
\tilde{a}_{K}
\end{array}\right]=\tilde{\xi}
$$

As the angular-velocity VKF_OT scheme, we introduce a weighting factor and combine (9) and (13), and then

$$
\begin{aligned}
& {\left[\begin{array}{c}
0 \\
0 \\
0 \\
\vdots \\
0 \\
\tilde{y}
\end{array}\right]-\left[\begin{array}{cccccc}
r \overleftrightarrow{M} & 0 & 0 & \cdots & 0 & 0 \\
0 & r \overleftrightarrow{M} & 0 & \cdots & 0 & 0 \\
0 & 0 & r \overleftrightarrow{M} & \cdots & 0 & 0 \\
\vdots & \vdots & \vdots & \vdots & \vdots & \vdots \\
0 & 0 & 0 & \cdots & 0 & r \overleftrightarrow{M} \\
\widetilde{B}_{1} & \widetilde{B}_{2} & \widetilde{B}_{3} & \cdots & \widetilde{B}_{k-1} & \widetilde{B}_{k}
\end{array}\right]\left[\begin{array}{c}
\tilde{a}_{1} \\
\tilde{a}_{2} \\
\tilde{a}_{3} \\
\vdots \\
\tilde{a}_{K-1} \\
\tilde{a}_{K}
\end{array}\right]} \\
& =\left[\begin{array}{c}
r \overleftrightarrow{Z} \\
\xi
\end{array}\right] .
\end{aligned}
$$


Equation (14) can be symbolized as

$$
\overleftrightarrow{Y}-\overleftrightarrow{P} \overleftrightarrow{A}=\overleftrightarrow{E}
$$

The evaluation of tracked order components is exactly to find a vector $\overleftrightarrow{A}$ fulfilling

$$
\min _{\overleftrightarrow{A}}\left(\|\overleftrightarrow{E}\|^{2}\right)=\min _{\overleftrightarrow{A}}\left(\overleftrightarrow{E}^{H} \overleftrightarrow{E}\right)=\min _{\overleftrightarrow{A}}(J)
$$

that is, $\partial J / \partial \overleftrightarrow{A}=0$. The vector $\overleftrightarrow{A}$ can be written as

$$
\overleftrightarrow{P}^{H} \overleftrightarrow{P} \overleftrightarrow{A}=\overleftrightarrow{P}^{H} \overleftrightarrow{Y}
$$

The matrix $\overleftrightarrow{P}^{H} \overleftrightarrow{P}$ is written as

$$
\overleftrightarrow{P}^{H} \overleftrightarrow{P}=\left[\begin{array}{ccccc}
\overleftrightarrow{S}_{B} & \overleftrightarrow{B}_{1,2} & \overleftrightarrow{B}_{1,3} & \ldots & \overleftrightarrow{B}_{1, K} \\
\overleftrightarrow{B}_{2,1} & \overleftrightarrow{S}_{B} & \overleftrightarrow{B}_{2,3} & \ldots & \overleftrightarrow{B}_{2, K} \\
\overleftrightarrow{B}_{3,1} & \overleftrightarrow{B}_{1,2} & \overleftrightarrow{S}^{\prime} & \ldots & \overleftrightarrow{B}_{3, K} \\
\vdots & \vdots & \vdots & \ddots & \vdots \\
\overleftrightarrow{B}_{K, 1} & \overleftrightarrow{B}_{K, 2} & \overleftrightarrow{B}_{K, 3} & \cdots & \overleftrightarrow{S}
\end{array}\right]
$$

where $\overleftrightarrow{S}=r^{2} \overleftrightarrow{M} \overleftrightarrow{M}^{T} \overleftrightarrow{I}$ and $\overleftrightarrow{B}_{u, v}=B_{u}^{H} B_{v}$. Moreover, $\overleftrightarrow{P}^{H}$ is written as

$$
\overleftrightarrow{P}^{H}=\left[\begin{array}{lllll}
\overleftrightarrow{B}_{1} & \overleftrightarrow{B}_{2} & \overleftrightarrow{B}_{3} & \ldots & \overleftarrow{B}_{K}
\end{array}\right]^{T}
$$

where $\overleftrightarrow{B}_{K}$ is the complex conjugate of $\overleftrightarrow{B}_{K}$.

\section{Principle of the Presented Algorithm}

As mentioned above, SPWVD has a promising timefrequency resolution. Therefore, we obtain frequencies and durations of modes from SPWVD distributions of testing guided waves. Furthermore, VKF_OT is adapted to realize isolation of different wave packages with obtained mode frequencies. Finally, the final mode waveforms are cut out from the wave packages of modes by durations of modes. The processing steps of the extension algorithm are shown in Figure 1 and are as follows.

(1) Smoothed Pseudo Wigner-Ville Distribution. SPWVD is used for processing testing signal to get corresponding timefrequency distribution. And the promising time-frequency resolution of the method can lead to a high calculation accuracy of frequencies and durations of modes.

(2) Extracting and Separating Valuable Areas of the TimeFrequency Panel. To extract and separate different modes, we do the following. (1) sum coefficients of each frequencyindexed group, and the calculation formula is

$$
E(\Theta)=\sum_{n=1}^{N} \operatorname{SPW}_{s}(n, \Theta)
$$

where $N$ denotes the number of the time variables of the timefrequency panel. (2) Calculate minimal values and maximum values of summations. Lines of the minimal values in the time-frequency panel can separate the valuable areas. And the maximum value evaluates whether the energy of a mode is strong enough to be kept. (3) Conduct threshold processing for the maximum values. And the equation is defined as

$$
E(\Theta)_{\max }= \begin{cases}\text { keep } & E(\Theta)_{\max }>T_{0} \\ \text { discard } & E(\Theta)_{\max } \leq T_{0}\end{cases}
$$

where $E(\Theta)_{\max }$ means the maximum of $E(\Theta)$. $T_{0}$ denotes the threshold for sift valuable modes. A mode with a strong energy is more valuable for detecting the debonding defect, so a threshold process is introduced to improve calculation efficiency. Moreover, the noise can also be removed by this operation. After this step, the areas of the timefrequency panel corresponding to the significant modes can be extracted and separated. Moreover, corresponding frequencies at the maximum values are taken as the mode frequencies.

(3) Vold-Kalman Filter Order Tracking. The mode frequencies have been calculated in Step (2). So we employ VKF_OT to filter the specific mode waveform with these frequencies. The different modes $f_{0 i}(i=1,2, \ldots, M)$ can be preliminarily isolated, and $M$ is number of the maximum values obtained in Step (2).

(4) Peak-Track Algorithm. Conduct the peak-track algorithm for the significant areas of SPWVD to obtain the primary IA of different modes. The principle of the peak-track algorithm can be found in [30]. And then, the IAs of significant modes can be obtained.

(5) Constructing Filters in Time Domain. To remove calculation error of the mode waveform from result obtained in Step (3), we construct a corresponding filter in time domain based on the primary IAs of different modes obtained in Step (4). And the equation is as follows:

$$
A_{i}= \begin{cases}1 & A_{0 i}>T_{A} \\ 0 & A_{0 i} \leq T_{A}\end{cases}
$$

where $A_{i}$ is the value of the $i$ th filter in time domain and $A_{0 i}$ denote the primary IAs of the $i$ th modes. $T_{A}$ is a threshold to remove calculation error of the mode waveform. 


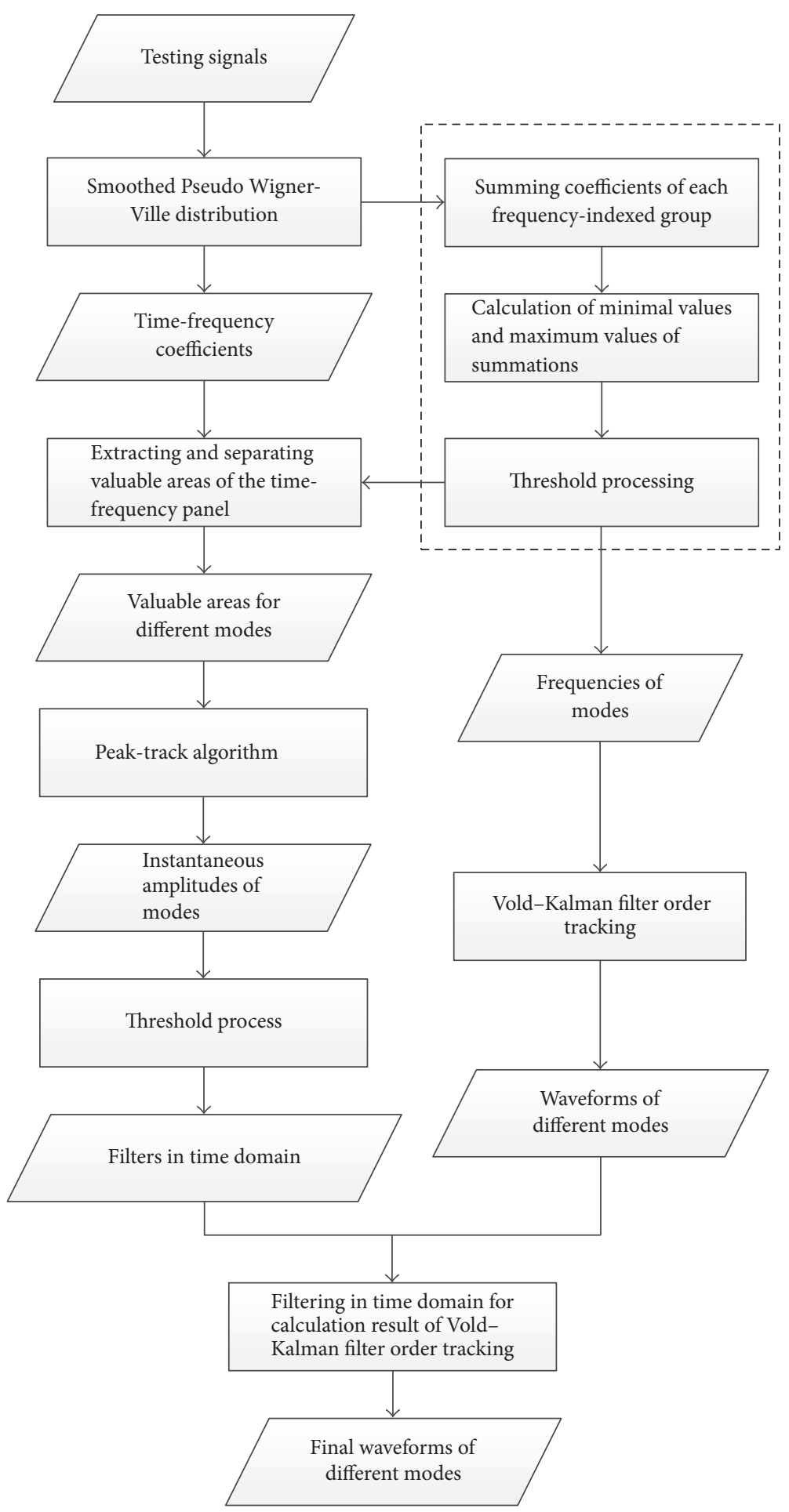

FIGURE 1: The different steps of the algorithm presented in the paper.

(6) Filtering in Time Domain for Calculation Result of Vold-Kalman Filter Order Tracking. Calculate the inner product between the mode waveforms obtained in Step (3) and the filter in time domain from Step (5). And then the final calculation result is obtained. The equation is

$$
f_{i}=A_{i} * f_{0 i} .
$$

In Section 4, the details of the present algorithm will be illustrated with a sample signal.

\section{Illustration of the Presented Algorithm}

We construct a sample signal to illustrate the presented algorithm, 


$$
\begin{aligned}
& s(t)=s_{1}(t)+s_{2}(t)+s_{3}(t), \\
& s_{1}(t)= \begin{cases}0 & 0 \leq t \leq 0.00005 \\
4000(t-0.00005) \sin (300000 \times 2 \pi t) & 0.00005<t \leq 0.0003 \\
-4000(t-0.00055) \sin (300000 \times 2 \pi t) & 0.0003<t \leq 0.00055 \\
0, & 0.00055<t \leq 0.001\end{cases} \\
& s_{2}(t)= \begin{cases}0 & 0 \leq t \leq 0.00025 \\
4000(t-0.00025) \sin (55000 \times 2 \pi t) & 0.00025<t \leq 0.0005 \\
-4000(t-0.00075) \sin (55000 \times 2 \pi t) & 0.0005<t \leq 0.00075 \\
0, & 0.00075<t \leq 0.001\end{cases} \\
& s_{3}(t)= \begin{cases}0 & 0 \leq t \leq 0.00045 \\
4000(t-0.00025) \sin (50000 \times 2 \pi t) & 0.00045<t \leq 0.0007 \\
-4000(t-0.00075) \sin (50000 \times 2 \pi t) & 0.0007<t \leq 0.00095 \\
0, & 0.00095<t \leq 0.001 .\end{cases}
\end{aligned}
$$

The sampling frequency is $2 \mathrm{MHz}$. The sample signal consists of three modes at frequencies of 50,55 , and $300 \mathrm{kHz}$. The curve of the sample signal in time domain is shown in Figure 2.

Firstly, we employ SPWVD for the sample signal to obtain the corresponding time-frequency panel, which is shown in Figure 3. As can be seen in Figure 3, the time resolution and frequency resolution of the time-frequency are promising. Besides, no interference is in the representation. However, the difference between the component at $50 \mathrm{kHz}$ and the component at $55 \mathrm{kHz}$ is so little that the two components cannot be separated in the SPWVD distribution of the sample signal, so is in Fourier spectrum, as shown in Figure 4.

After that, (20) is used on the SPWVD distribution. As shown in Figure 5, three maximum values at 50, 55, and $300 \mathrm{kHz}$ are kept. So VKF_OT is employed on the sample signal with 50,55 , and $300 \mathrm{kHz}$ to get the corresponding filtering results.

And then, the primary IAs of different modes are obtained by the peak-track algorithm. The filters in time domain of different modes are obtained by employing (22) on the primary IAs, as shown in Figure 6. We can adjust the threshold in (22) to get filters in time domain of different modes with a high accuracy in time resolution.

Finally, we conduct time-domain filter for the result of VKF_OT, and the results are shown in Figure 7. As presented in Figure 7, the decomposition results almost overlap with the corresponding original modes. To further validate the effectiveness of the presented algorithm, we calculate the error of the decomposition result, as shown in Figure 8. The absolute error is less than 0.1 for the modes at 50 and $55 \mathrm{kHz}$ and is less than 0.02 for the mode at $300 \mathrm{kHz}$, which reveals that the calculation accuracy of the presented algorithm is promising.

To compare with EEMD, the sample signal is also processed by this decomposition method. Figure 9 shows the coefficients of correlation between different IMFs and the sample signal. We can learn that IMFs 1-4 are vital components of the signal, as the coefficients of correlation are relatively greater. So these IMFs are shown in Figure 10. It is visible that the mode mixing occurs in EEMD for modes at 50 and $55 \mathrm{kHz}$, as shown in Figure 10(b). Because the ratio between modes at 50 and $55 \mathrm{kHz}$ is greater than 0.75 , they cannot be separated, as revealed in [25]. Therefore, the poor frequency resolution is a limitation of EEMD for its application.

\section{Details of the Experimental}

The material of the specimen is a specific composite material. The size is $400 \mathrm{~mm} \times 300 \mathrm{~mm} \times 3 \mathrm{~mm}$ and contains 15 layers. The corresponding size diagram is presented in Figure 11. Two debonding defects are in the specimen. The side length of the defects is, respectively, $20 \mathrm{~mm}$ and $30 \mathrm{~mm}$.

Figure 12 is the diagram of the testing principle. In testing process, the exciting probe sends an exciting wave, and then the receiving probe will receive excited lamb waves. The excitation wave is a sine wave with a frequency of $100 \mathrm{kHz}$. The sampling frequency is $20 \mathrm{MHz}$. Three sets of signal are collected in the experiment, responding to three situations, that is, no defect, $20 \mathrm{~mm}$ defect, and $30 \mathrm{~mm}$ defect, respectively. The corresponding GWs collected in the experiment are presented in Figure 13. 


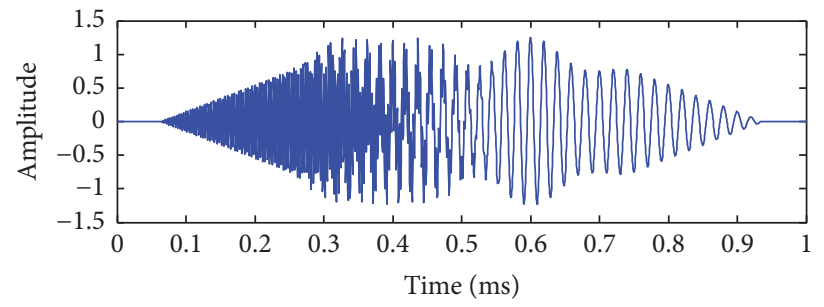

Figure 2: The curve of the sample signal in time domain.

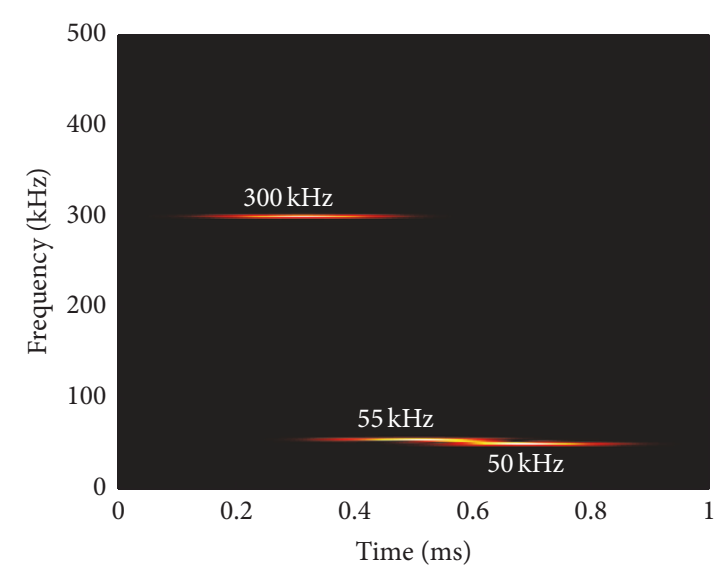

(a)

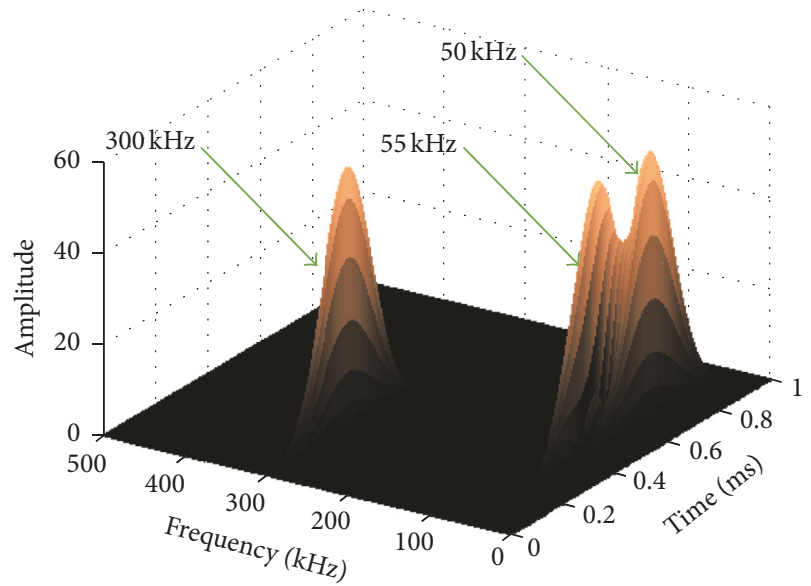

(b)

FIGURE 3: The representation of the sample signal by using SPWVD.

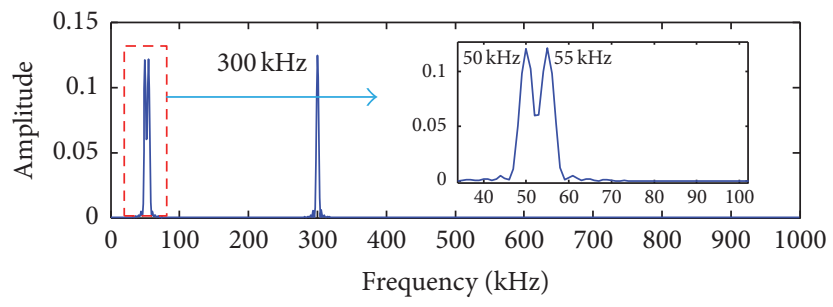

Figure 4: The Fourier spectrum of the sample signal.

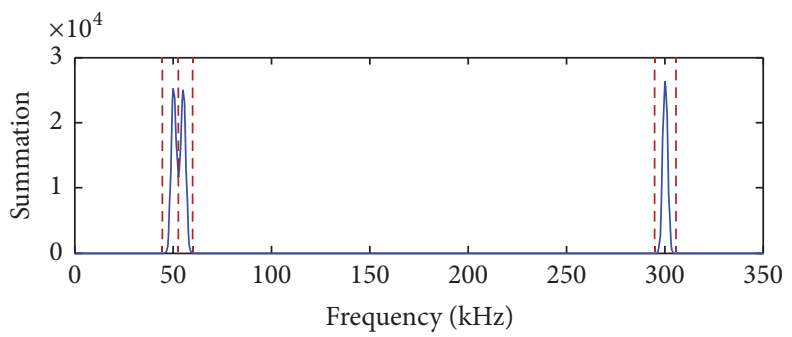

FIGURE 5: The different frequency-group summations of the time-frequency panel of the sample signal.

\section{Result and Discussion}

Figure 15 presents the decomposition result of experimental signals by using the presented algorithm. As shown in Figures 14 and 15, two modes exist in the experimental signal of no defect and are at $50.3 \mathrm{kHz}$ and $34.5 \mathrm{kHz}$. The signal of $20 \mathrm{~mm}$ defect consists of modes at $49.2 \mathrm{kHz}, 41.2 \mathrm{kHz}$, and $34.1 \mathrm{kHz}$. And the signal of $30 \mathrm{~mm}$ defect consists of modes at $49.8 \mathrm{kHz}$, $44.8 \mathrm{kHz}$, and $33.9 \mathrm{kHz}$. Figure 16 shows these modes are decomposed by the presented algorithm. This indicates the 


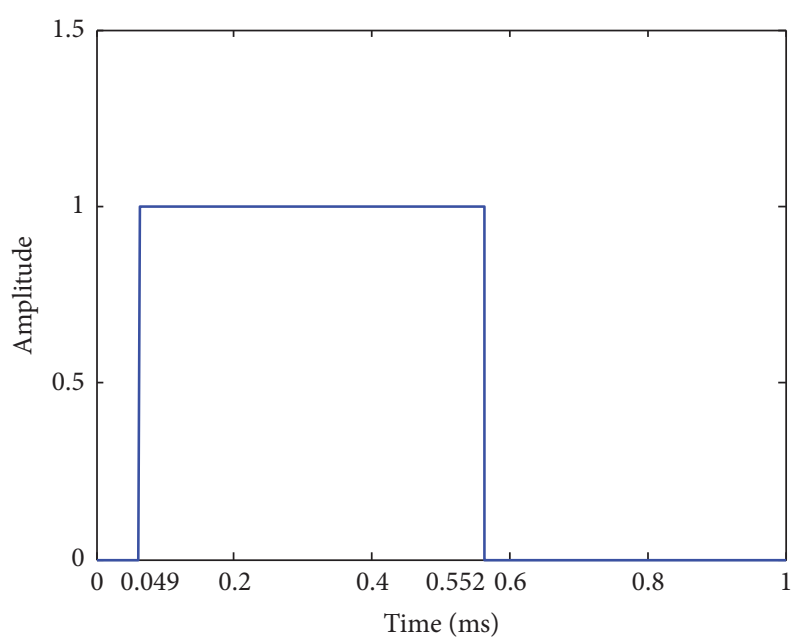

(a)

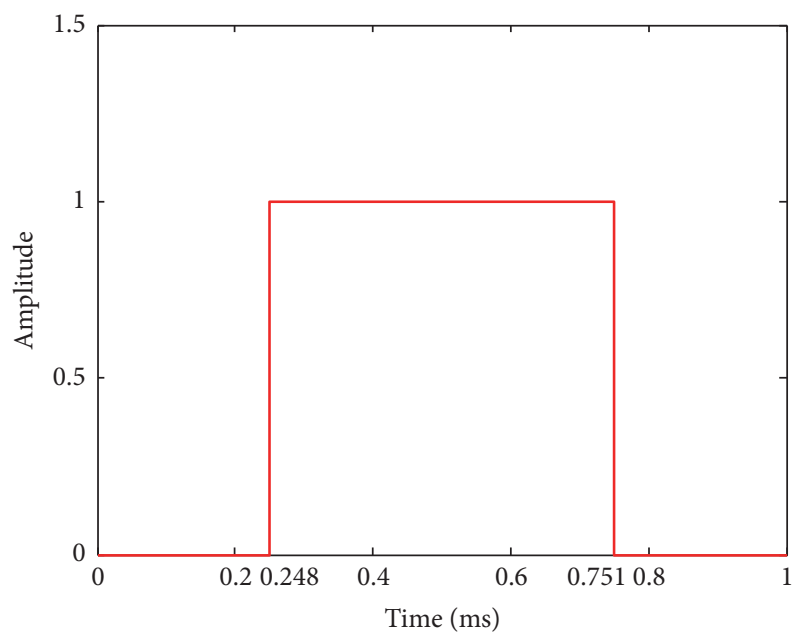

(b)

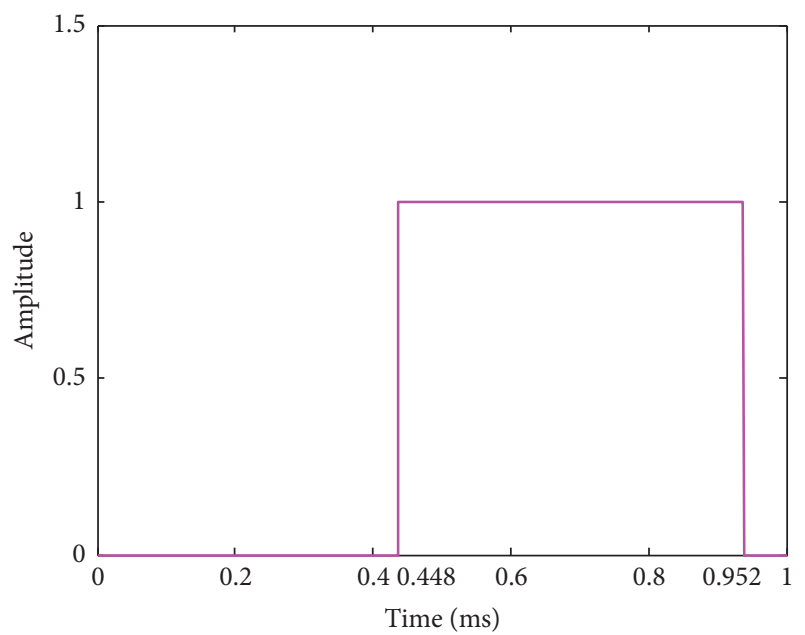

(c)

FIGURE 6: The filters in time domain of different modes: (a) mode at $300 \mathrm{kHz}$, (b) mode at $55 \mathrm{kHz}$, and (c) mode at $50 \mathrm{kHz}$.

method is effective in isolating different modes. Besides, considering that $50.3 \mathrm{kHz}, 49.2 \mathrm{kHz}$, and $49.8 \mathrm{kHz}$ pose little

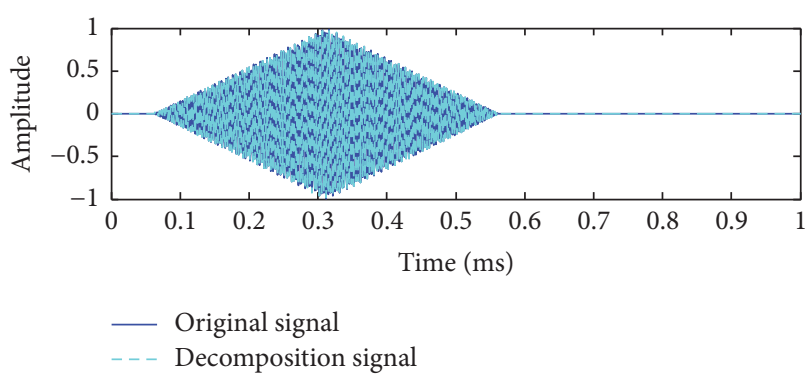

(a)

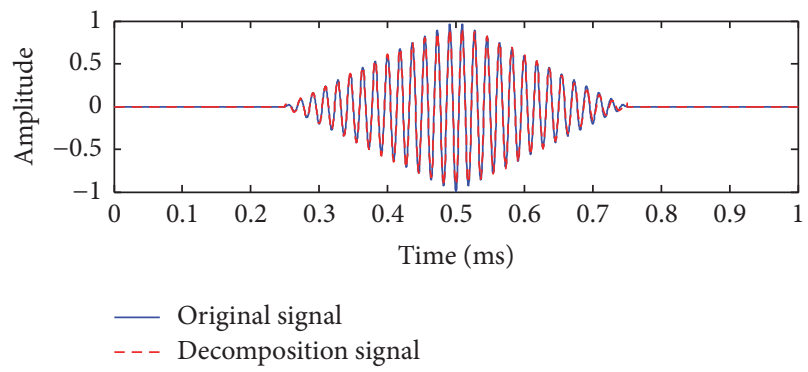

(b)

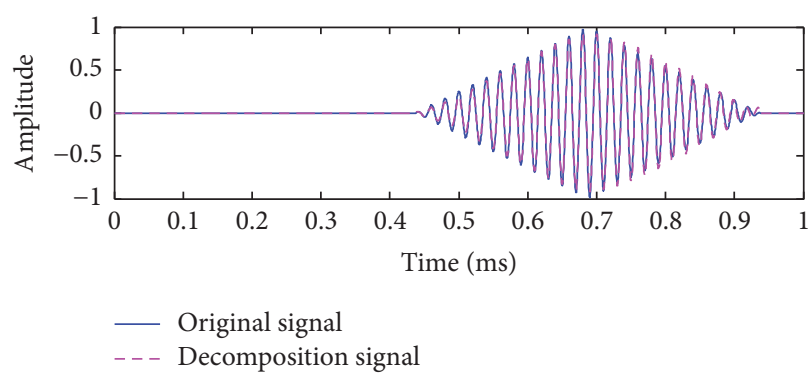

(c)

FIGURE 7: The decomposition result and the original modes of the sample signal $x$ by the presented algorithm: (a) mode at $300 \mathrm{kHz}$, (b) mode at $55 \mathrm{kHz}$, and (c) mode at $50 \mathrm{kHz}$.

differences and this phenomenon is similar to $34.5 \mathrm{kHz}$, $34.1 \mathrm{kHz}$, and $33.9 \mathrm{kHz}$, it seems that defects stimulate new modes and $41.2 \mathrm{kHz}$ for $20 \mathrm{~mm}$ defect and $44.8 \mathrm{kHz}$ for $30 \mathrm{~mm}$ defect. On the basis of this phenomenon, we can try to detect the defect. Moreover, the frequency of the new mode becomes greater along with the defect size $(41.2 \mathrm{kHz}$ for $20 \mathrm{~mm}$ and $44.8 \mathrm{kHz}$ for $30 \mathrm{~mm}$ ). Maybe we can try to evaluate the size of the defect, according to this relationship. Finally, as we have isolated different wave packets, the location of defect can be obtained by the decomposition results.

\section{Conclusion}

This paper presents a decomposition algorithm aiming to analyze the characteristics of ultrasonic GWs generated in a NDT for the debonding in a type of composite material by combining SPWVD and VKF_OT. The presented method succeeds in isolating different GW modes. On the basis of 


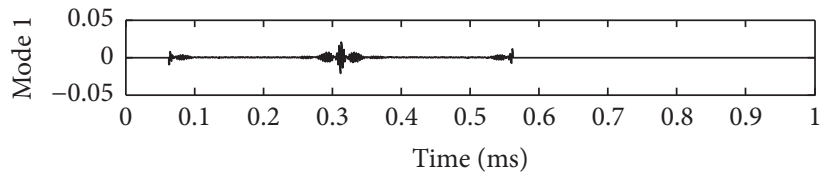

(a)

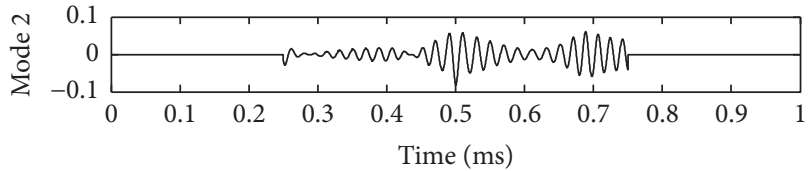

(b)

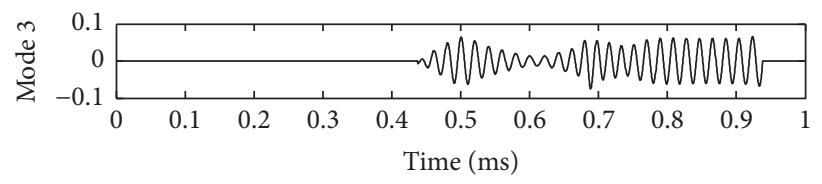

(c)

FIGURE 8: The errors of decomposition result of the sample signal $x$ by the presented algorithm: (a) mode at $300 \mathrm{kHz}$, (b) mode at $55 \mathrm{kHz}$, and (c) mode at $50 \mathrm{kHz}$.

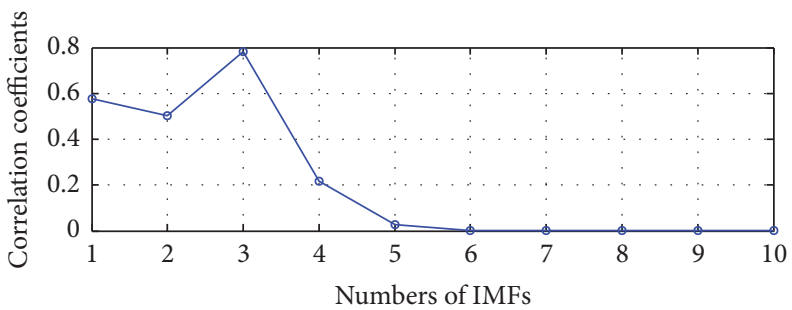

FIGURE 9: Correlation coefficients between IMFs and the original signal.
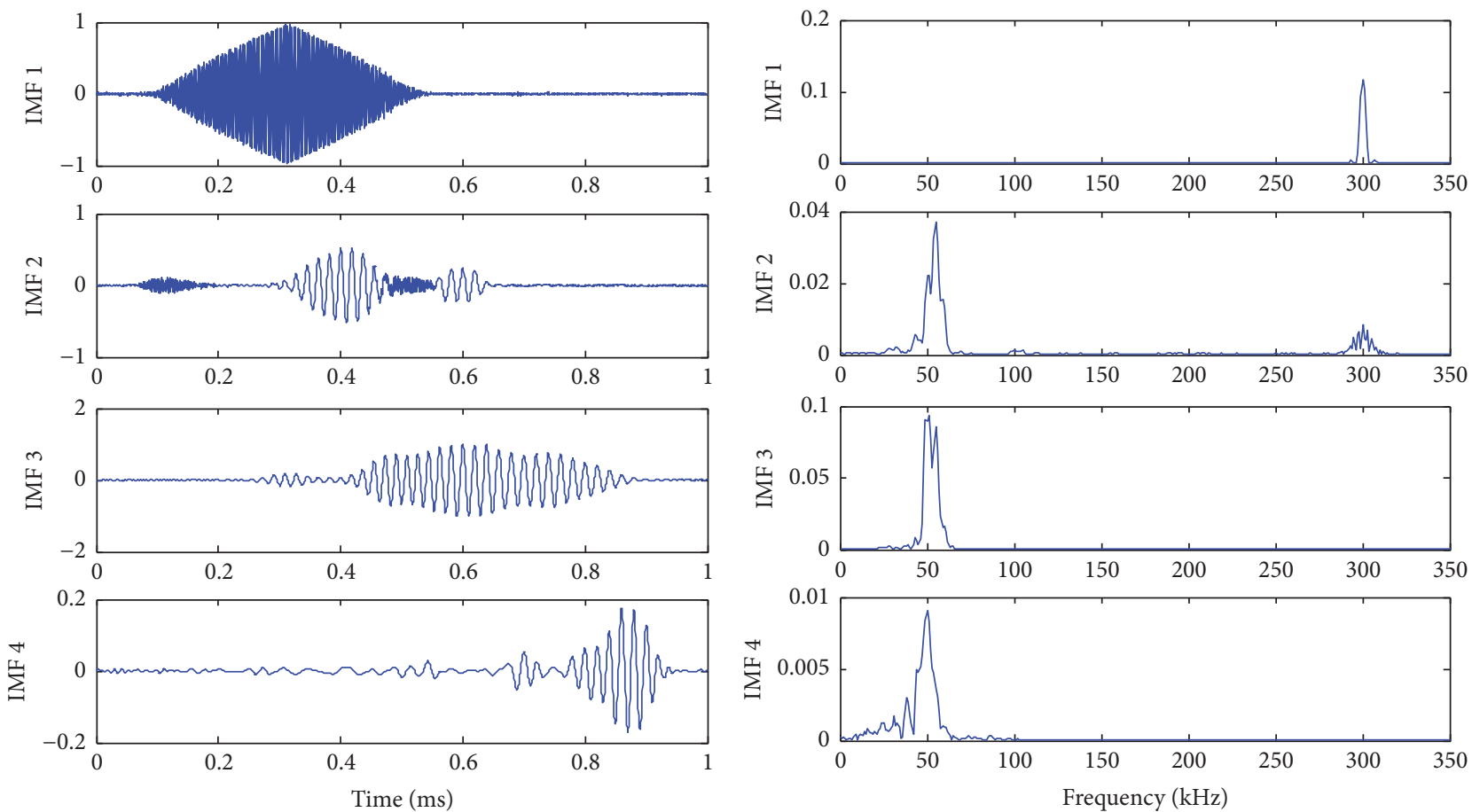

(a)

(b)

FIGURE 10: The IMFs 1-4 in the EEMD of the sample signal and the corresponding Fourier spectrums: (a) the IMFs and (b) the corresponding Fourier spectrums. 


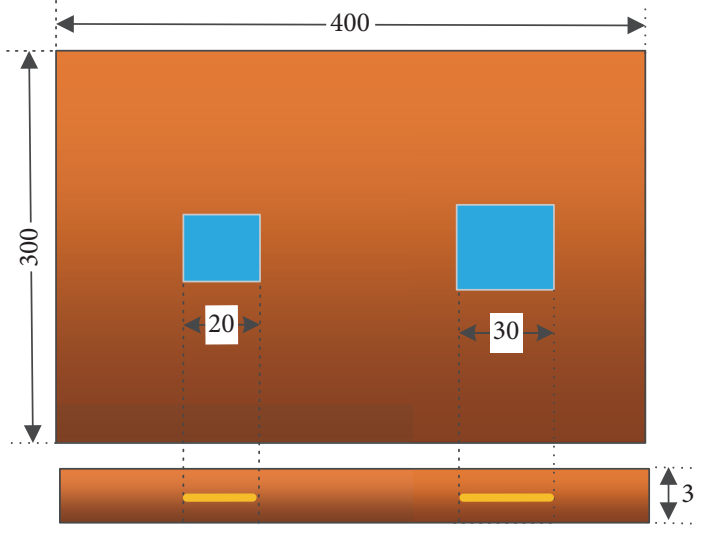

FIGURE 11: The size diagram of the specimen.

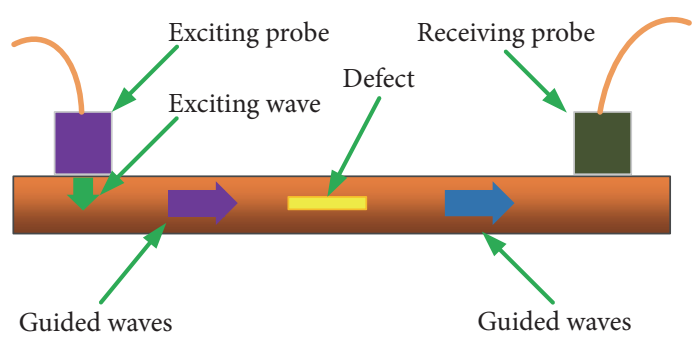

FIGURE 12: The diagram of the testing principle.

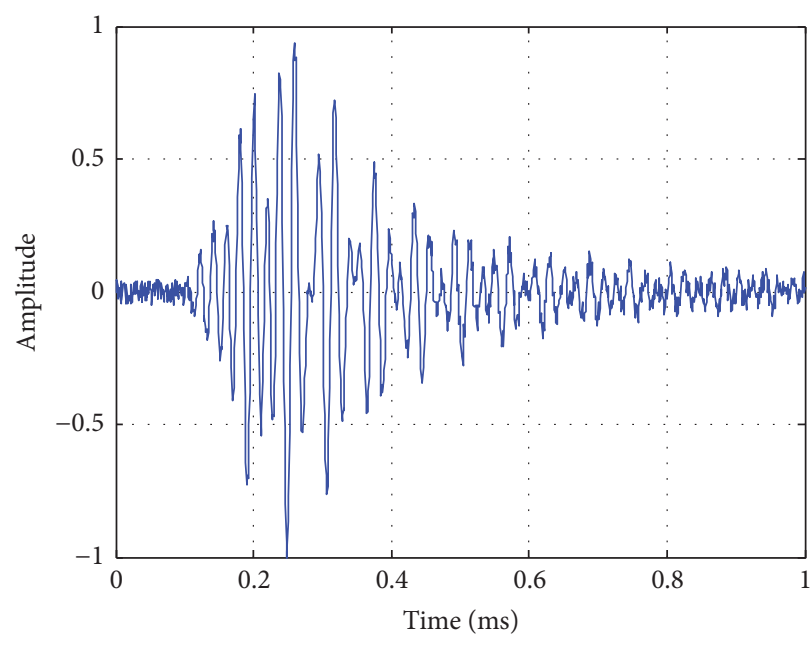

(a)

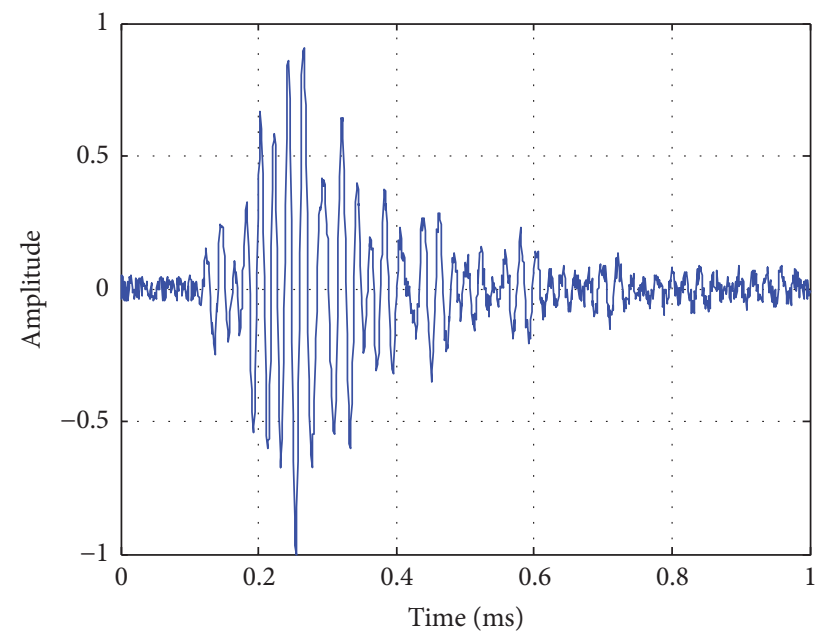

(b)

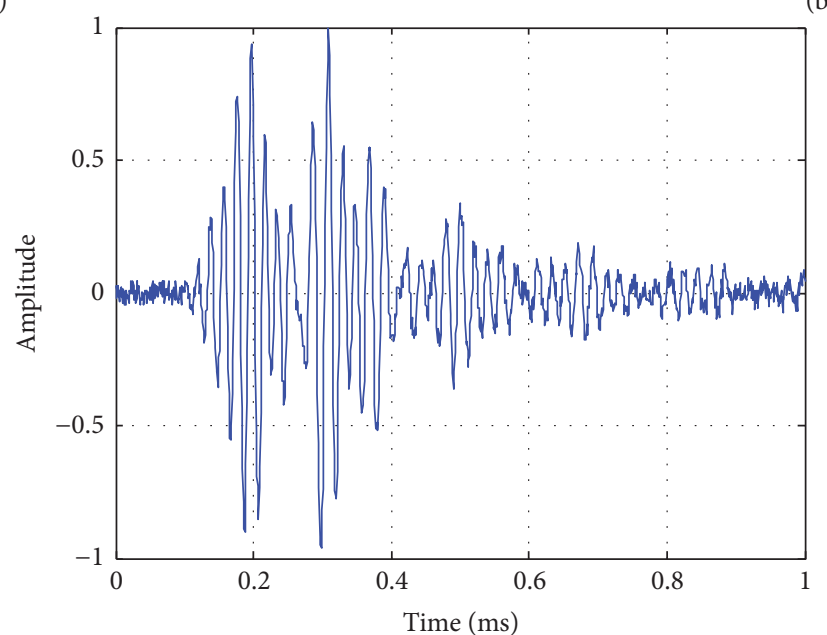

(c)

Figure 13: The GWs collected in the experiment: (a) no defect, (b) $20 \mathrm{~mm}$, and (c) $30 \mathrm{~mm}$. 


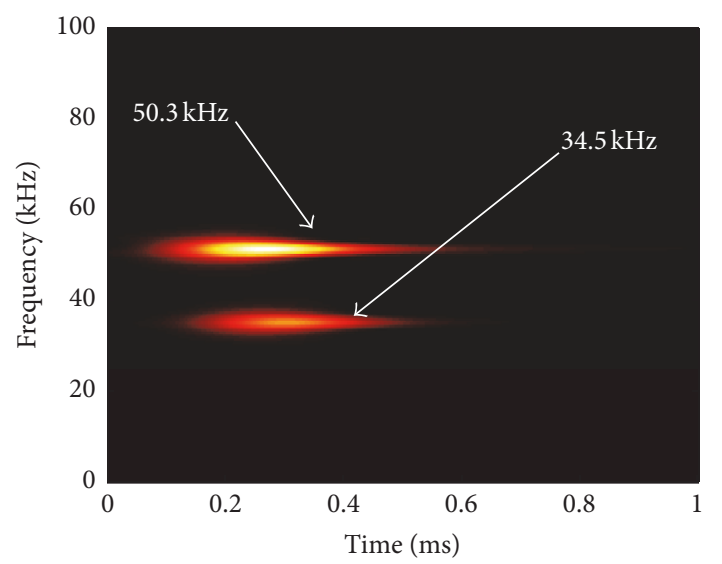

(a)

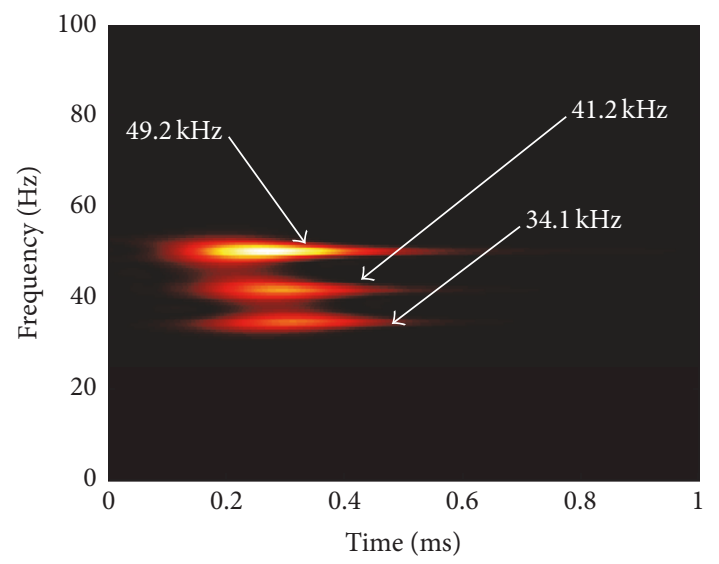

(b)

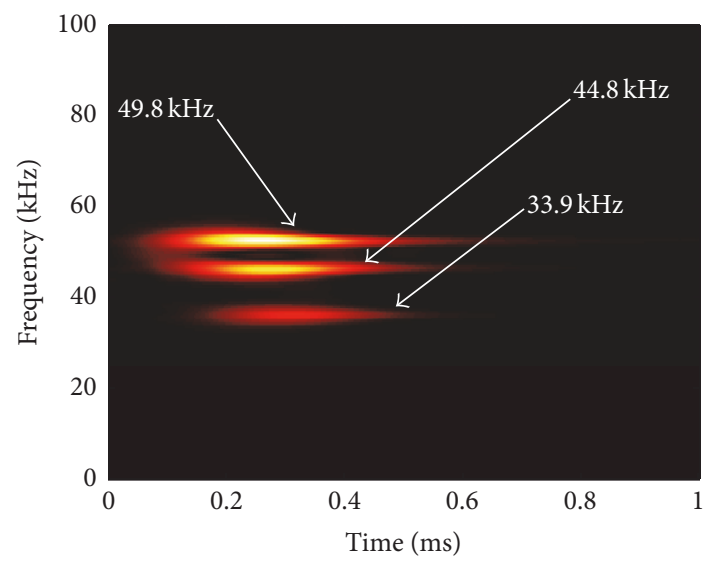

(c)

FIGURE 14: The SPWVD representations of the GWs in the experiment: (a) no defect, (b) $20 \mathrm{~mm}$, and (c) $30 \mathrm{~mm}$.

the presented algorithm, the characteristics of the experimental signals were investigated. Some conclusions, which are valuable for identification of defect, calculation of defect size, and locating defect, are obtained. The technique also can be applied in analogue NDTs and NDEs on the basic of the ultrasonic GWs. Further research will be done to validate the feasibility for locating defects by the algorithm.

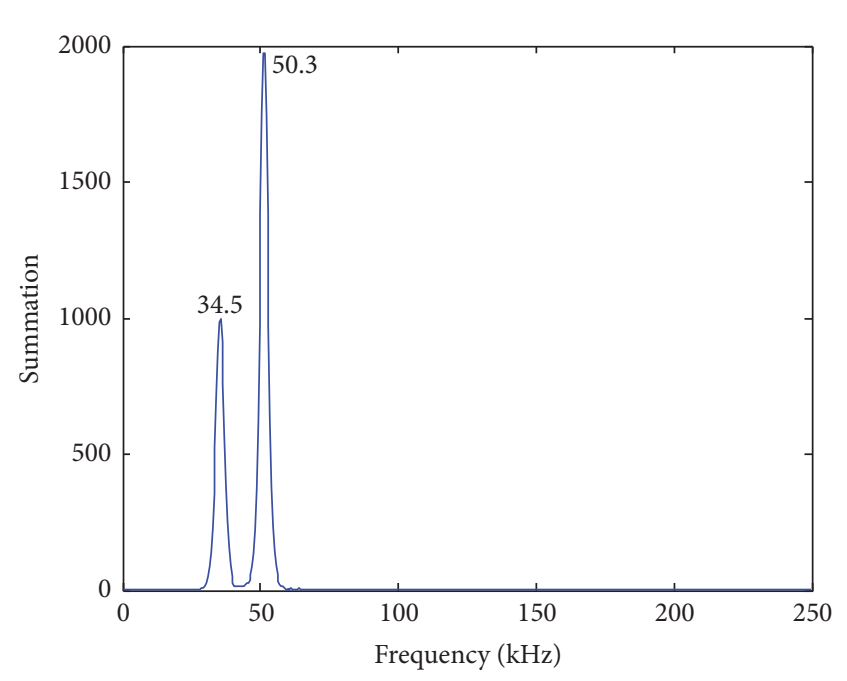

(a)

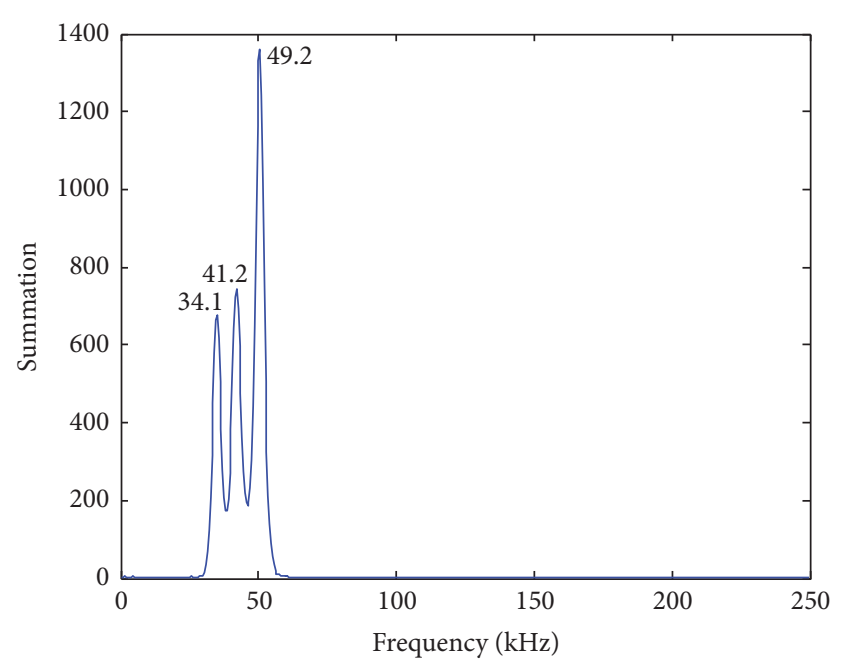

(b)

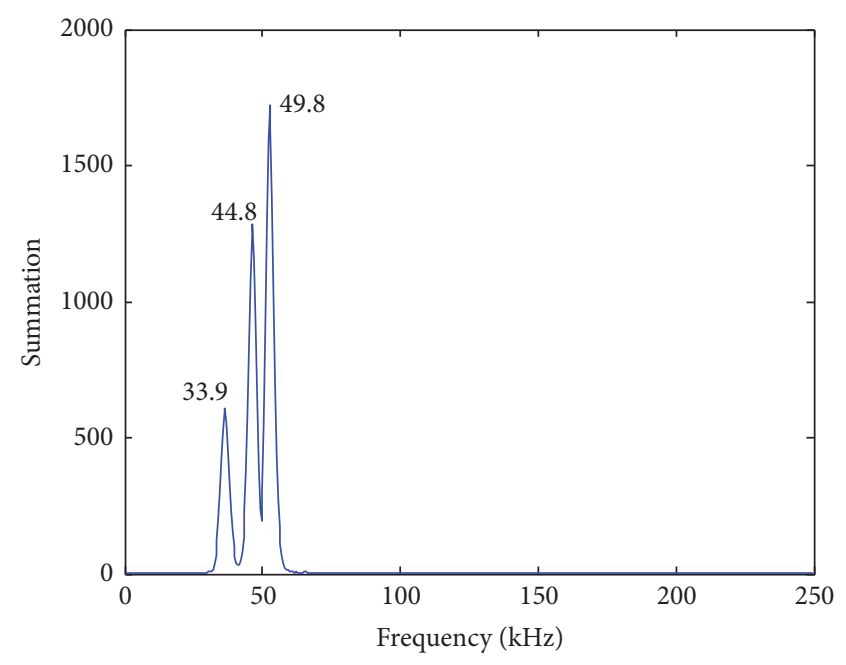

(c)

FIGURE 15: The different frequency-group summations of the timefrequency panel of the experimental signals: (a) no defect, (b) $20 \mathrm{~mm}$, and (c) $30 \mathrm{~mm}$. 

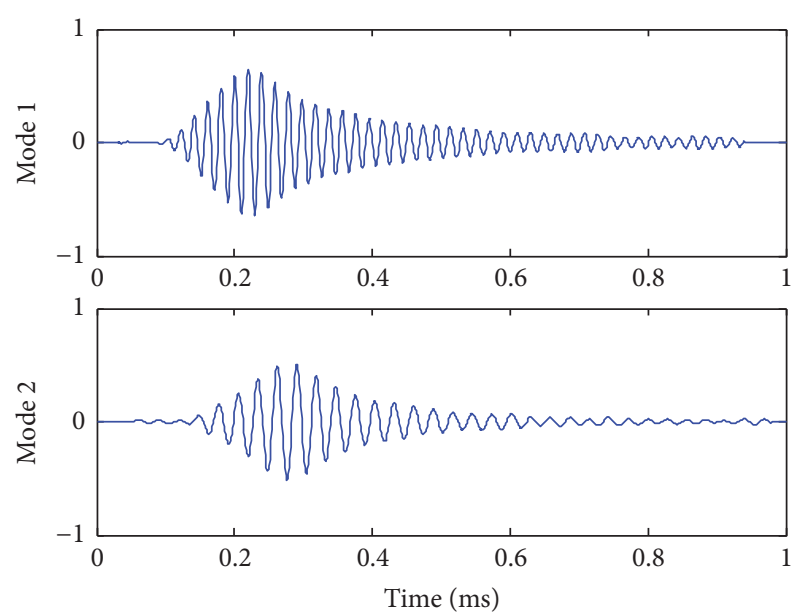

(a)
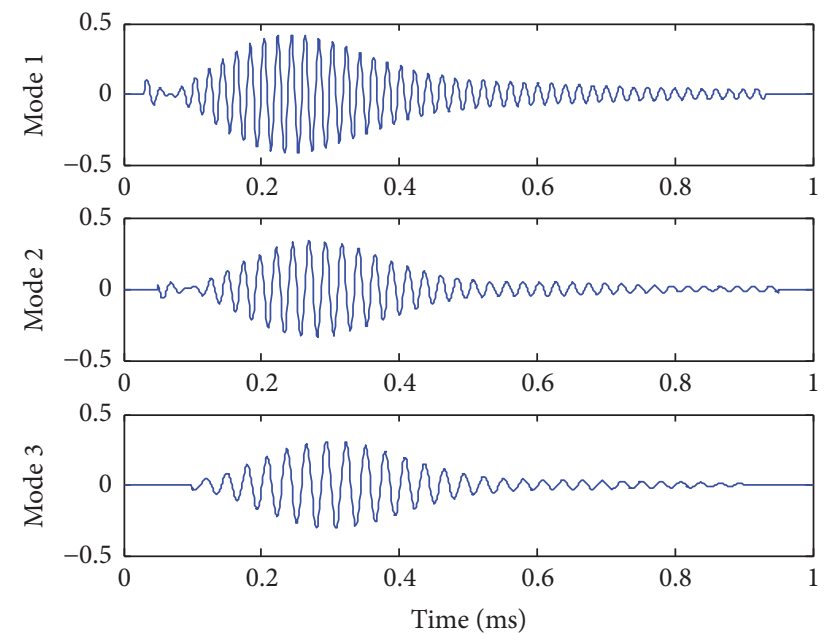

(b)
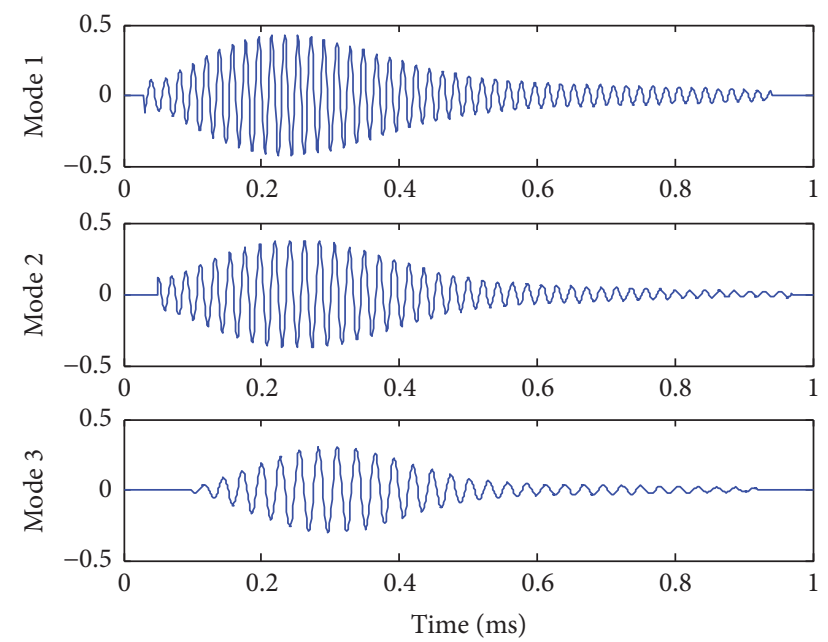

(c)

FIGURE 16: The decomposition result of experimental signals by using the presented algorithm: (a) no defect, (b) $20 \mathrm{~mm}$, and (c) $30 \mathrm{~mm}$.

\section{Conflicts of Interest}

The authors declare that they have no conflicts of interest.

\section{References}

[1] R. Zhou, Z. Li, and J. Sun, "Crack deflection and interface debonding in composite materials elucidated by the configuration force theory," Composites Part B: Engineering, vol. 42, no. 7, pp. 1999-2003, 2011.

[2] T. Liu, W. Zhang, and S. Yan, "A novel image enhancement algorithm based on stationary wavelet transform for infrared thermography to the de-bonding defect in solid rocket motors," Mechanical Systems and Signal Processing, vol. 62, no. 10, pp. 366-380, 2015.

[3] J. Li, Y. Lu, R. Guan, and W. Qu, "Guided waves for debonding identification in CFRP-reinforced concrete beams," Construction and Building Materials, vol. 131, pp. 388-399, 2017.

[4] J. Wu, Z. Ma, and Y. Zhang, "A Time-Frequency Research for Ultrasonic Guided Wave Generated from the Debonding Based on a Novel Time-Frequency Analysis Technique," Shock and Vibration, vol. 2017, Article ID 5686984, 2017.

[5] L. Longbiao, "Modeling the Effect of Interface Wear on Fatigue Hysteresis Behavior of Carbon Fiber-Reinforced CeramicMatrix Composites," Applied Composite Materials, vol. 22, no. 6, pp. 887-920, 2015.

[6] S. Khare, M. Razdan, P. Munshi, B. V. S. Sekhar, and K. Balasubramaniam, "Defect detection in carbon-fiber composites using lamb-wave tomographic methods," Research in Nondestructive Evaluation, vol. 18, no. 2, pp. 101-119, 2007.

[7] K. S. Tan, N. Guo, B. S. Wong, and C. G. Tui, "Experimental evaluation of delaminations in composite plates by the use of Lamb waves," Composites Science and Technology, vol. 53, no. 1, pp. 77-84, 1995.

[8] S. W. Kercel, M. B. Klein, and B. Pouet, "Bayesian separation of Lamb wave signatures in laser ultrasonics," in Proceedings of the Applications and Science of Computational Intelligence III, vol. 4055 of Proceedings of SPIE, pp. 350-361, April 2000.

[9] J. Cai, L. Shi, and X. P. Qing, "A time-distance domain transform method for Lamb wave dispersion compensation considering signal waveform correction," Smart Materials and Structures, vol. 22, no. 10, Article ID 105024, 2013.

[10] P. Rizzo and F. L. di Scalea, "Feature extraction for defect detection in strands by guided ultrasonic waves," Structural Health Monitoring, vol. 5, no. 3, pp. 297-308, 2006.

[11] R. Gangadharan, C. R. L. Murthy, S. Gopalakrishnan, and M. R. Bhat, "Time reversal technique for health monitoring of metallic structure using Lamb waves," Ultrasonics, vol. 49, no. 8, pp. 696-705, 2009.

[12] T. Liu, S. Yan, and W. Zhang, "Time-frequency analysis of nonstationary vibration signals for deployable structures by using the constant-Q nonstationary gabor transform," Mechanical Systems and Signal Processing, vol. 75, pp. 228-244, 2016.

[13] W. Wu, G. Jiang, S. Huang, and C. J. Leo, "Vertical dynamic response of pile embedded in layered transversely isotropic soil," Mathematical Problems in Engineering, Article ID 126916, 12 pages, 2014.

[14] H. Li, X. Deng, and H. Dai, "Structural damage detection using the combination method of EMD and wavelet analysis," Mechanical Systems and Signal Processing, vol. 21, no. 1, pp. 298306, 2007.

[15] C. A. Paget, S. Grondel, and K. Levin, "Damage assessment in composites by Lamb waves and wavelet coefficients," Smart Materials and Structures, vol. 12, no. 3, pp. 393-412, 2003.

[16] L. Yu, J. Bao, and V. Giurgiutiu, "Signal processing techniques for damage detection with piezoelectric wafer active sensors 
and embedded ultrasonic structural radar," in Proceedings of the Smart Structures and Materials 2004 - Sensors and Smart Structures Technologies for Civil, Mechanical, and Aerospace Systems, pp. 492-503, USA, March 2004.

[17] Y. Y. Kim and E.-H. Kim, "Effectiveness of the continuous wavelet transform in the analysis of some dispersive elastic waves," The Journal of the Acoustical Society of America, vol. 110, no. 1, pp. 86-94, 2001.

[18] D. Yu, J. Cheng, and Y. Yang, "Application of EMD method and Hilbert spectrum to the fault diagnosis of roller bearings," Mechanical Systems and Signal Processing, vol. 19, no. 2, pp. 259270, 2005.

[19] L. Yu, S. Wang, and K. K. Lai, "Forecasting crude oil price with an EMD-based neural network ensemble learning paradigm," Energy Economics, vol. 30, no. 5, pp. 2623-2635, 2008.

[20] R. Osegueda, V. Kreinovich, S. Nazarian, and E. Roldan, "Detection of cracks at rivet holes in thin plates using Lambwave scanning," in Proceedings of the International Society for Optical Engineering: Smart Nondestructive Evaluation and Health Monitoring of Structural and Biological Systems II, pp. 5566, USA, March 2003.

[21] Y. Yu and C. Junsheng, "A roller bearing fault diagnosis method based on EMD energy entropy and ANN," Journal of Sound and Vibration, vol. 294, no. 1-2, pp. 269-277, 2006.

[22] L. Salvino, A. Purekar, and D. J. Pines, "Health monitoring of 2D plates using EMD and hilbert phase," in Proceedings of the 4th International Workshop on Structural Health Monitoring, Stanford University, Calif, USA, 2005.

[23] Z. H. Guo, W. G. Zhao, H. Y. Lu, and J. Wang, "Multistep forecasting for wind speed using a modified EMD-based artificial neural network model," Renewable Energy, vol. 37, no. 1, pp. 241-249, 2012.

[24] A. Y. Fu, W. Liu, and X. Deng, "Detecting phishing web pages with visual similarity assessment based on Earth Mover's Distance (EMD)," IEEE Transactions on Dependable and Secure Computing, vol. 3, no. 4, pp. 301-311, 2006.

[25] G. Rilling and P. Flandrin, "One or two frequencies? The empirical mode decomposition answers," IEEE Transactions on Signal Processing, vol. 56, no. 1, pp. 85-95, 2008.

[26] H. Vold and J. Leuridan, "High resolution order tracking at extreme slew rates, using kalman tracking filters," SAE Technical Papers, 1993.

[27] H. Vold, H. Herlufsen, M. Mains, and D. Corwin-Renner, "Multi axle order tracking with the Vold-Kalman tracking filter," S V Sound and Vibration, vol. 31, no. 5, pp. 30-34, 1997.

[28] P. Flandrin and B. Escudié, "An interpretation of the PseudoWigner-Ville distribution," Signal Processing, vol. 6, no. 1, pp. 2736, 1984.

[29] M.-C. Pan, W.-C. Chu, and D.-D. Le, "Adaptive angular-velocity Vold-Kalman filter order tracking-Theoretical basis, numerical implementation and parameter investigation," Mechanical Systems and Signal Processing, vol. 81, pp. 148-161, 2016.

[30] Z. Chen and R. C. Maher, "Semi-automatic classification of bird vocalizations using spectral peak tracks," The Journal of the Acoustical Society of America, vol. 120, no. 5, pp. 2974-2984, 2006. 


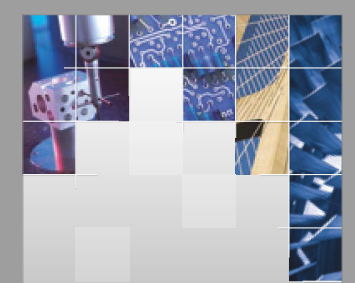

\section{Enfincering}
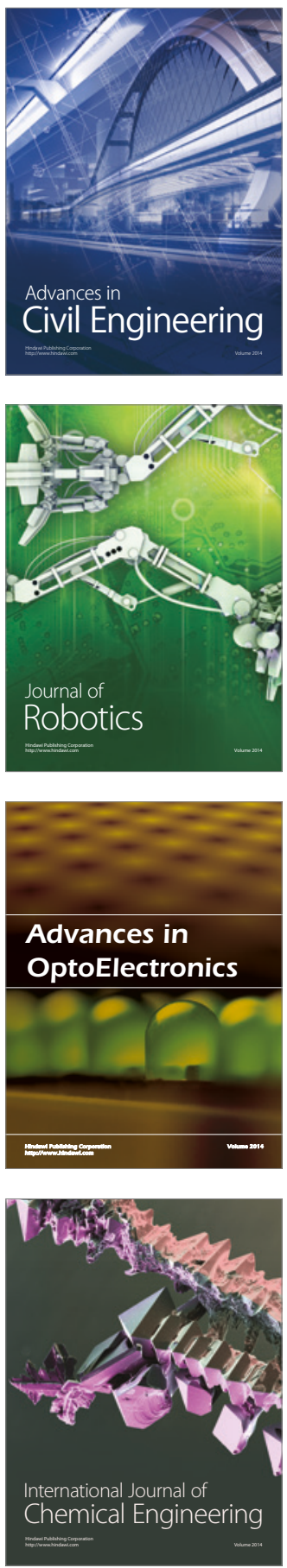

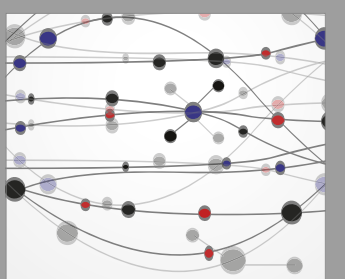

The Scientific World Journal

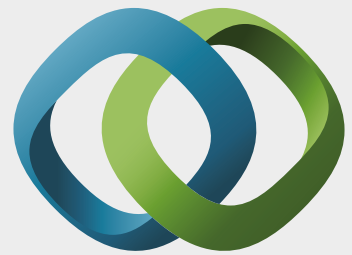

\section{Hindawi}

Submit your manuscripts at

https://www.hindawi.com
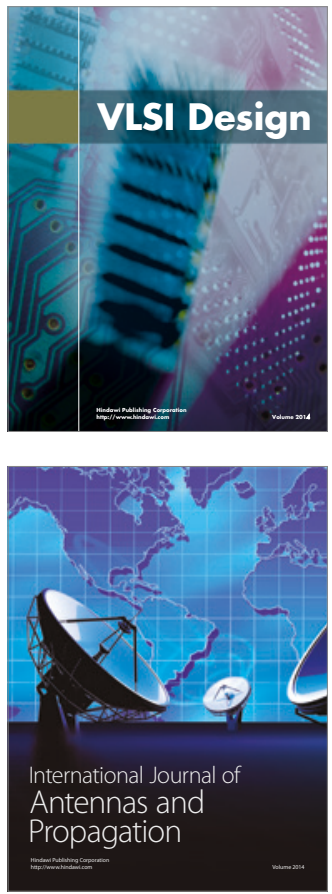

\section{Rotating}

Machinery
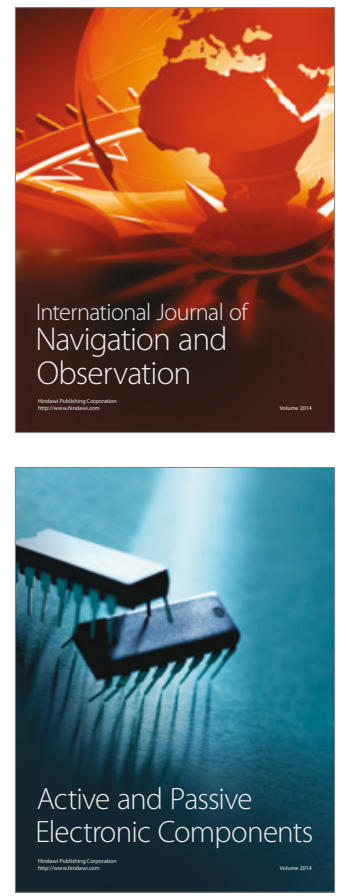
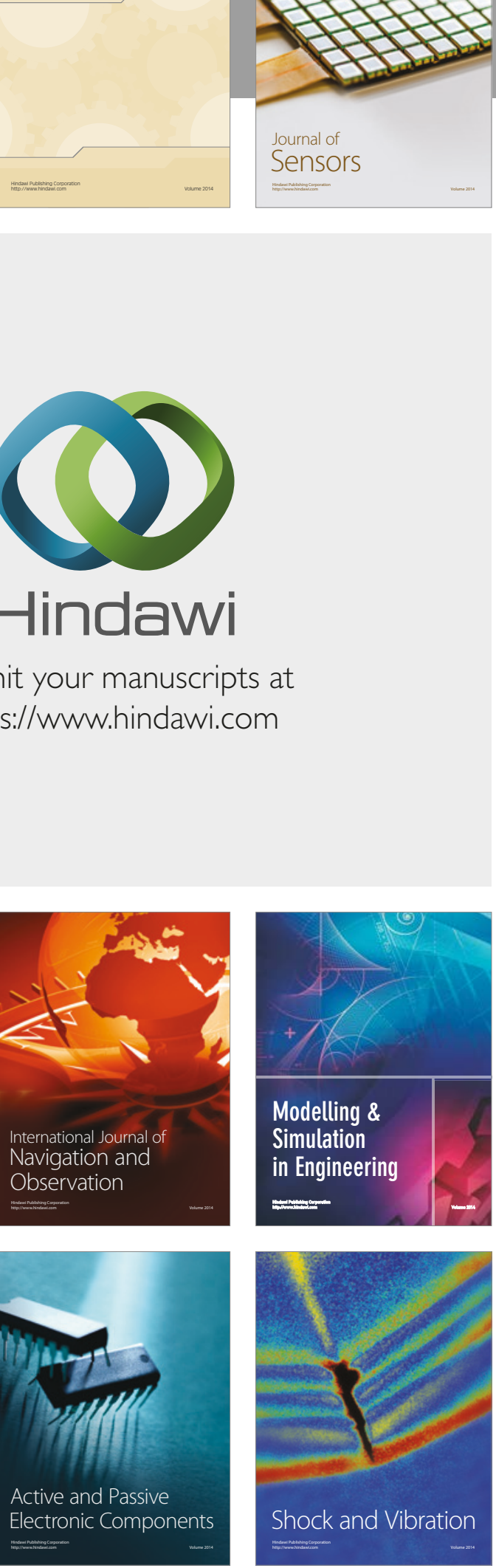
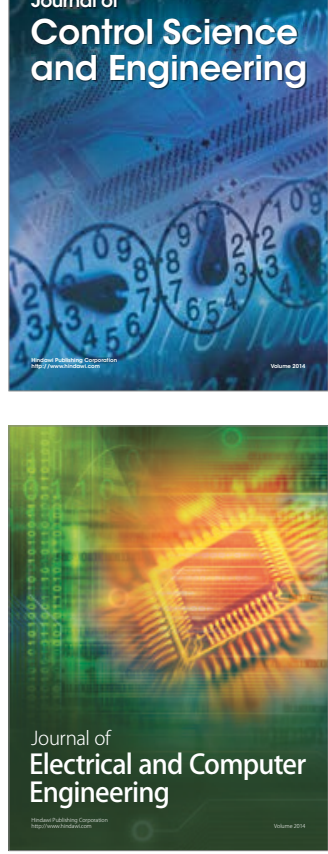

Distributed

Journal of

Control Science

and Engineering
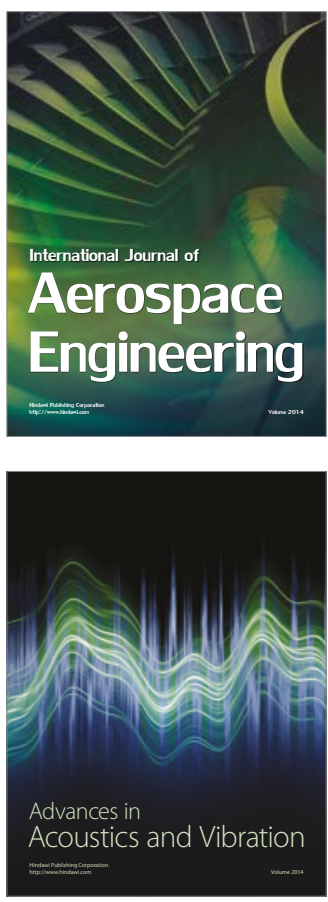

Sensor Networks 